\section{Pinguecula and contact lenses}

\begin{abstract}
Purpose To assess the relationship between age and the incidence and severity (determined by a grading system) of pinguecula in contact lens (CL) wearers, and to compare the grade of pinguecula between $C L$ wearers and non-wearers.

Methods A total of $600 \mathrm{CL}$ wearers (94 wore hard CLs (HCLs) and 506 wore soft CLs (SCLs)) aged 11-60 years and 579 non-wearers aged 10-60 years were enrolled. The age, gender, medical history, ocular history, and grade of pinguecula at two locations (nasal and temporal) were determined in all subjects. Results There was an age-related increase in the grade of pinguecula among both CL wearers and non-wearers. The grade of pinguecula at the temporal conjunctiva was higher in CL wearers than in non-wearers $(P=0.01907)$, whereas it was higher in HCL wearers than SCL wearers at both the nasal and temporal conjunctiva $(P<0.00001$ and $P<0.00001)$.

Conclusions This was the first assessment of the severity of pinguecula in a large consecutive series of CL wearers. Our results suggest that the use of CLs is an important risk factor for pinguecula.
\end{abstract}

Eye (2010) 24, 1685-1691; doi:10.1038/eye.2010.120; published online 10 September 2010

Keywords: pinguecula; grading; contact lens

\section{Introduction}

Pinguecula is one of the most common age-related eye diseases and is characterized by the appearance of yellowish to brown nodules on the bulbar conjunctiva near the sclerocorneal junction. The development of pinguecula is associated with aging. ${ }^{1-6}$

We previously examined the incidence of conjunctivochalasis in a hospital-based population $^{7}$ and in contact lens (CL) wearers. ${ }^{8}$ We found that the prevalence and severity of
T Mimura', T Usui', M Mori², H Yamamoto', H Obata ${ }^{3}$, S Yamagami', H Funatsu' ${ }^{4}, \mathrm{H} \mathrm{Noma}^{4}$, $\mathrm{N} \mathrm{Honda}{ }^{1}$ and S Amano ${ }^{1}$

conjunctivochalasis were dependent on both the age $^{7}$ of the subjects and the duration of wearing CLs. ${ }^{8}$ The grade of conjunctivochalasis was highest in hard CL (HCL) wearers, followed by soft CL (SCL) wearers, and then non-wearers. ${ }^{9}$ Wearing CLs can cause chronic conjunctival inflammation due to hypoxia, mechanical friction, dehydration, or incompatibility with the lens material. ${ }^{9}$ However, there have been no large-scale surveys that have investigated pinguecula in CL wearers. On the other hand, tear film instability because of CLs may be involved in the pathogenesis of pinguecula. It may be of benefit to CL wearers to know whether CLs are a risk factor for developing pinguecula. Accordingly, this study was performed to (1) assess the relationship between wearing CLs and pinguecula, (2) introduce a new pinguecula grading system, and (3) compare the severity of pinguecula between HCL wearers and SCL wearers by using our new grading system.

This report covers our recent work on the relationship between wearing CLs and the occurrence of conjunctivochalasis. ${ }^{9}$ Therefore, the study population consisted of the same subjects reported in a previous paper. $^{9}$

\section{Materials and methods}

\section{Subject}

This was a prospective, cross-sectional, and consecutive case series. This study was performed in accordance with the Helsinki Declaration of 1975 and its 1983 revision. Institutional Review Board approval was obtained and informed consent was also obtained from each subject. Consecutive CL wearers attending our outpatient clinic for the periodic eye examinations between January and June 2007 were enrolled in the study. Almost all of the patients lived in Tokyo or Kanagawa in Japan. Patients with a history of ocular surgery or punctal occlusion, and those with infectious conjunctivitis, inflammatory conjunctivitis, proptosis, or eyelid abnormalities (such as
${ }^{1}$ Department of Ophthalmology, University of Tokyo Graduate School of Medicine, Tokyo, Japan

${ }^{2}$ Department of Ophthalmology, Toranomon Hospital, Tokyo, Japan

${ }^{3}$ Department of Ophthalmology, Jichi Medical University, Tochigi, Japan

${ }^{4}$ Department of Ophthalmology, Yachiyo Medical Center, Tokyo Women's Medical University, Chiba, Japan

Correspondence: T Mimura, Department of Ophthalmology, University of Tokyo Graduate School of Medicine, 7-3-1 Hongo, Bunkyo-ku, Tokyo, 113-8655 Japan. Tel: + 81-3-3815-5411 (Ex33503);

Fax: + 81-3-3817-0798

E-mail: mimurat-tky@ umin.ac.jp

Received: 9 August 2009 Accepted in revised form: 10 July 2010

Published online: 10 September 2010 
entropion, ectropion, and trichiasis) were excluded. Moreover, patients using both HCLs and SCLs were excluded so that the differences between HCL and SCL wearers could be investigated. Consequently, the study population consisted of 506 SCL wearers and $94 \mathrm{HCL}$ wearers, including 237 males and 363 females aged $28.9 \pm 11.3$ years (mean \pm SD), with an age range of $11-60$ years (Table 1). The type of HCL was all rigid gas permeable lens, but not polymethylmethacrylate lens. The common reasons for the choice of HCL were the high myopia with astigmatism, a low cost of maintenance compared with SCL, and the parents' experience with HCLs. All CL wearers were under the age 60 years. First, 1034 patients were selected from among 1992 non-wearers, who attended our outpatient clinic for eye screening tests between January and June 2007 by excluding patients of 60 years of age and older in order to match for age with CL wearers. Next, we selected 564 age-matched controls in each age group from among 1034 control subjects by stratified random sampling using a random number table on the computer list. Thus, each decade age group was matched by using a computer database. Controls lived in the same geographic area as the CL wearers.

\section{Grading system for pinguecula}

Pinguecula was graded into three categories as follows: grade $\mathrm{P}(0)=$ none, grade $\mathrm{P}(1)=$ mild or moderate pinguecula, and grade $\mathrm{P}(2)=$ severe pinguecula (Figure 1). Grading was carried out separately for lesions affecting the temporal and nasal halves of the conjunctiva. The diagnosis and grading of pingueculae was carried out by two skilled optometrists and an ophthalmologist (TM). The graders were trained by scoring 30 standardized sample photographs of the eyes. After training, the three graders recorded scores (3-point scale) of 120 pinguecula located on both the nasal and temporal conjunctiva in 60 eyes of 30 subjects, before the study started. A high degree of consistency among the scores of three graders was obtained $(94.2 \%)$. They were aware of the CL status of all subjects. We evaluated the grade of pinguecula immediately after CL removal. Although data were obtained for both eyes, only data

Table 1 Clinical profile of the subjects

\begin{tabular}{lccc}
\hline & Contact lens wearers & Non-wearers & P-value \\
\hline $\begin{array}{l}\text { Number of patients } \\
\text { Age (years) }\end{array}$ & 600 & 564 & - \\
Sex & $28.9 \pm 11.3$ & $29.6 \pm 13.4$ & NS \\
$\quad$ Male & & & \\
Female & $237(39.5 \%)$ & $276(48.9 \%)$ & $288(51.1 \%)$ \\
Refraction & $363(60.5 \%)$ & & $<0.0001$ \\
Spherical equivalent (D) & & $-2.47 \pm 2.61$ & $<0.0001$ \\
Sphere (D) & $-4.86 \pm 2.47$ & $-2.17 \pm 2.55$ & NS \\
Cylinder (D) & $-4.52 \pm 2.41$ & $-0.70 \pm 0.71$ & \\
\hline
\end{tabular}

Abbreviations: D, diopters; NS, not significant.

Values are expressed as mean \pm s.d. and were compared with unpaired Student's $t$-test.

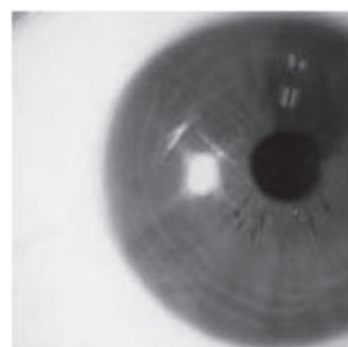

Grade $\mathbf{P}(0)$

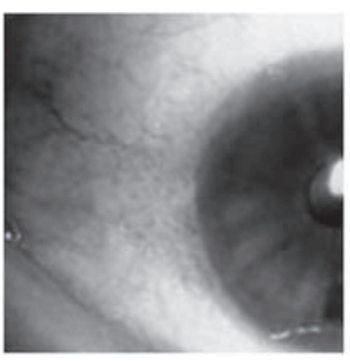

Grade $P(1)$

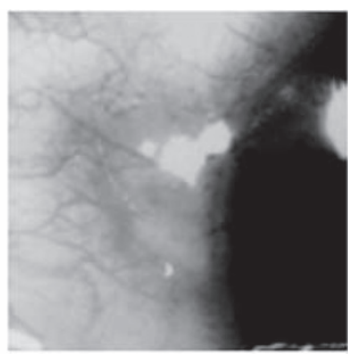

Grade $\mathbf{P ( 2 )}$

Figure 1 Pinguecula grading system. Pinguecula was graded into three categories as follows: grade $\mathrm{P}(0)=$ no pinguecula, grade $\mathrm{P}(1)=$ mild, or moderate pinguecula (yellowish-white and flat or slightly elevated lesion), and grade $\mathrm{P}(2)=$ severe pinguecula (highly vascular and elevated lesion). 
from the right eye were used for analysis. Any discrepancies among the three graders were adjudicated by an independent senior doctor.

\section{Statistical analysis}

The paired or unpaired Student's $t$-test was used to compare the mean values between the two groups and the $\chi^{2}$-test was used to compare percentages. Relations among the variables were investigated by calculating Pearson's correlation coefficients and partial correlation coefficients. Correlations were also evaluated by Fisher's Z-transformation method and Bartlett's test. Grades of pinguecula were compared between the two groups by the two-tailed Mann-Whitney $U$-test. The level of significance was set at $P<0.05$ for all analyses, which were carried out with the Stat View statistical software package (Abacus Concepts, Berkeley, CA, USA).

\section{Results}

Figure 2 shows the grade of pinguecula in each age group. As shown in Figure 2, the prevalence of pinguecula located on both the nasal and temporal conjunctiva increased dramatically with age among both CL wearers (11-20 years, 2.6\% (nasal) and 3.2\% (temporal), 21-30 years; 15.6 and $18.0 \%, 31-40$ years, 23.0 and $25.7 \%, 41-50$ years, 46.2 and $50.8 \%, 51-60$ years, 77.4 and $80.6 \%$, and total subjects $11-60$ years, 20.4 and $22.6 \%$ ) and non-wearers (11-20 years, $0.0 \%$ (nasal) and $0.6 \%$ (temporal), 21-30 years; 5.2 and 5.7\%, 31-40 years, 7.4 and $7.4 \%, 41-50$ years, 49.3 and $47.8 \%, 51-60$ years, 49.2 and $52.5 \%$, and total subjects $11-60$ years, 13.8 and $14.4 \%$ ). There was no significant difference in the grade of pinguecula between the nasal and the temporal conjunctiva in each age group. However, the grade of pinguecula was significantly higher in CL wearers than in non-wearers (mean grade for the nasal conjunctiva: $0.27 \pm 0.57$ vs $0.18 \pm 0.47, P=0.00173$; temporal conjunctiva: $0.29 \pm 0.56$ vs $0.19 \pm 0.48, P=0.00100$, unpaired $t$-test), as was the prevalence of pinguecula (nasal conjunctiva: $P=0.06203$; temporal conjunctiva: $P=0.01907$, two-tailed Mann-Whitney $U$-test)

(Figure 2).

The location of pinguecula was compared between CL wearers and non-wearers in each age group (Figure 3). In both group, most of the patients with pinguecula had pinguecula in the both nasal and temporal conjunctiva.

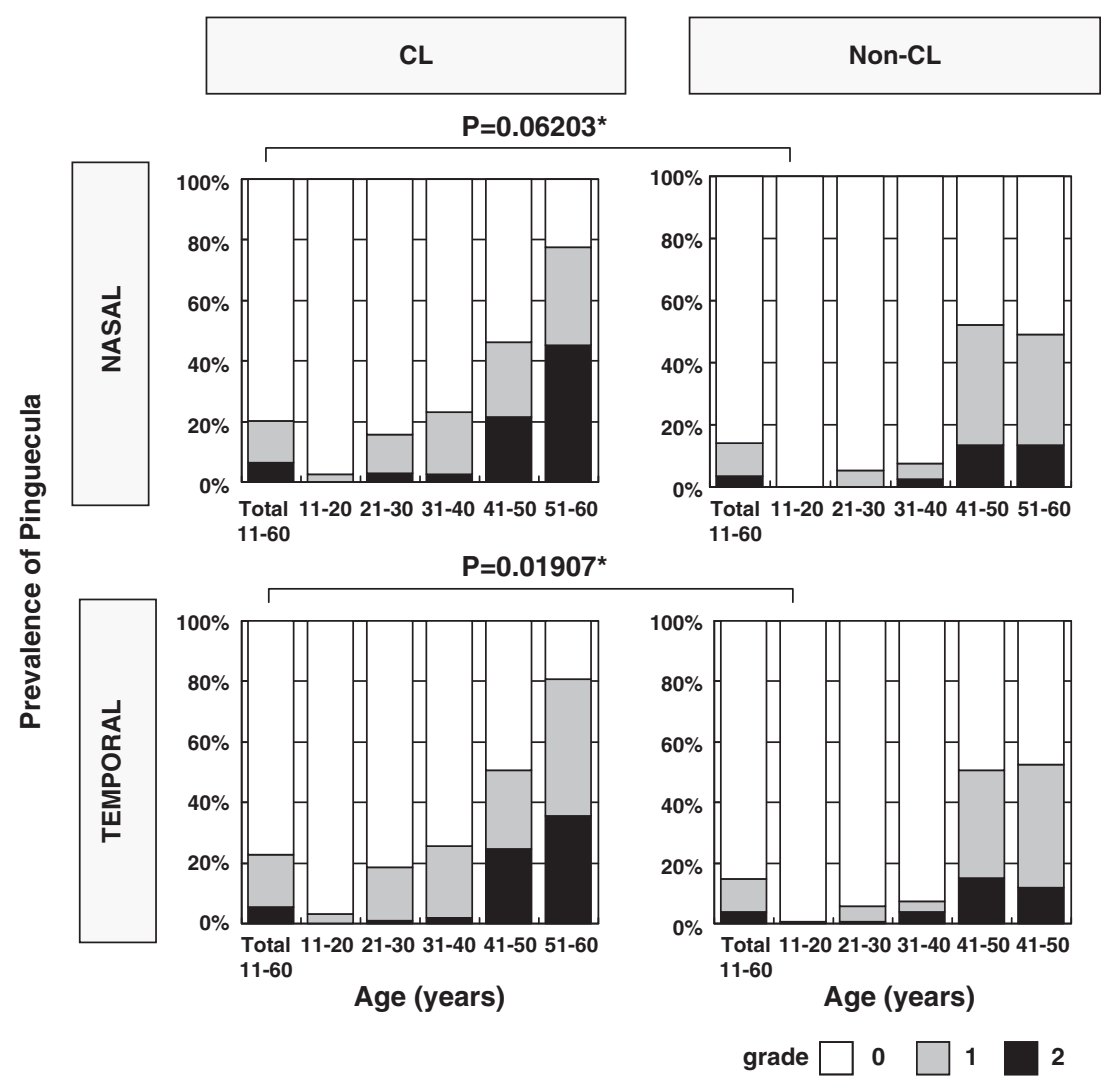

Figure 2 Comparison of the grade of pinguecula at the nasal and temporal areas of the conjunctiva in CL wearers and non-wearers. Pinguecula was graded according to the system indicated in Figure 1 and the Materials and methods. *Mann-Whitney $U$-test. 

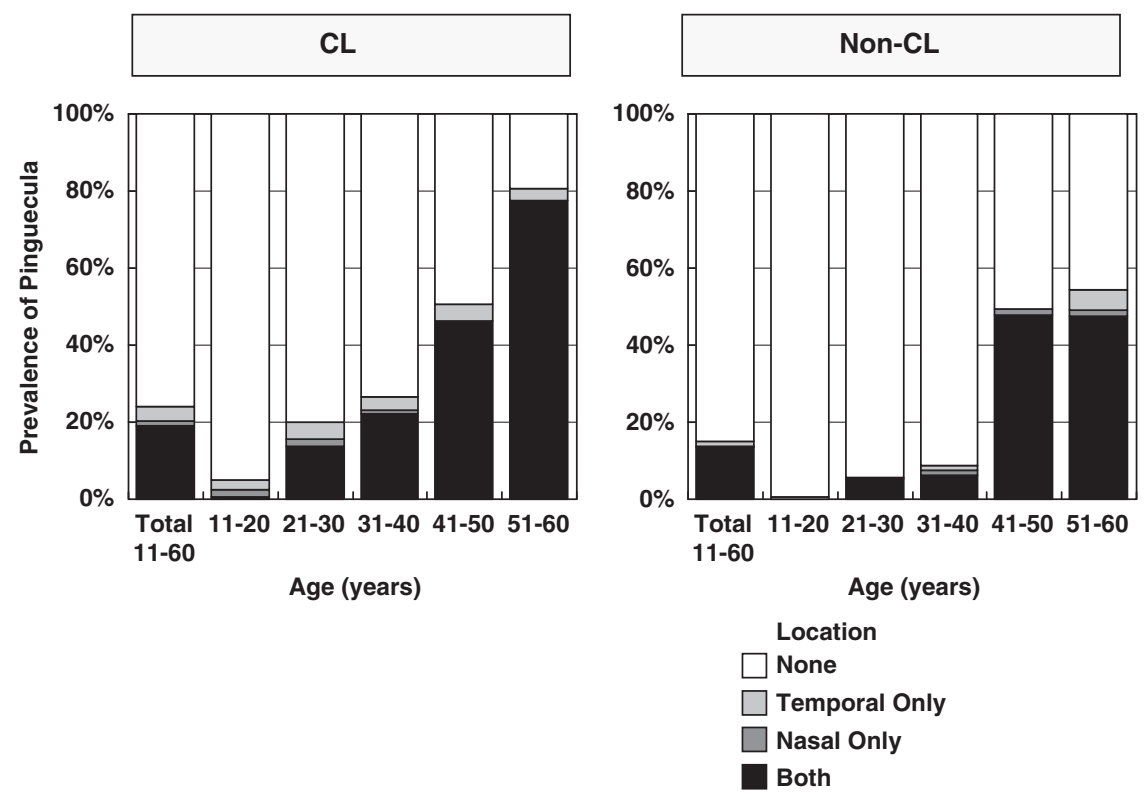

Figure 3 Comparison of the location of pinguecula in each age group between CL wearers and non-wearers. The location of pingueculal was classified into four categories: (1) no pinguecula, (2) temporal pinguecula only, (3) nasal pinguecula only, and (4) both temporal and nasal pinguecula.

There was no significant difference in the location of pinguecula between $\mathrm{CL}$ wearers and non-wearers in each age group (two-tailed Mann-Whitney $U$-test).

The grade of pinguecula was higher in HCL wearers than in SCL wearers at both the nasal and temporal conjunctiva $(P<0.00001$ and $P<0.00001$, respectively, two-tailed Mann-Whitney $U$-test) (Figures $4 a$ and b). There were significant differences in the prevalence and location of pinguecula between HCL and SCL wearers in some age groups $(P<0.05$, two-tailed Mann-Whitney $U$-test, Figure 5).

The grade of pinguecula was significantly correlated with the duration of CL wear in SCL (nasal; $r=0.222$, $P<0.00001$, and temporal; $r=0.237, P<0.00001)$ and HCL wearers (nasal; $r=0.554, P<0.00001$, and temporal; $r=0.506, P<0.00001)$.

\section{Discussion}

This study showed that both the prevalence and grade of pinguecula were higher in CL wearers than in nonwearers, and that the grade of pinguecula was higher in HCL wearers than SCL wearers. Although the prevalence of pinguecula is well known to increase with age, no previous attempt has made to assess the influence of wearing CLs on pinguecula. This study provides the first data about the grade of pinguecula in CL wearers.

The prevalence of pinguecula was very low during the first three decades of life and steadily increased after the fourth decade in non-wearers (Figure 2), indicating that pinguecula increases with age. Among CL wearers, however, pinguecula was even seen in younger patients, and its prevalence at both the nasal and temporal areas was significantly higher than in non-wearers (Figure 2). Constant friction and inflammation of the conjunctiva caused by the CL edge may be responsible for the younger onset of pinguecula in CL wearers.

Next, we compared the grade of pinguecula between HCL and SCL wearers, and showed that the grade was higher in HCL wearers than SCL wearers (Figures $4 \mathrm{a}$ and $b$ ). The duration of $\mathrm{CL}$ wearing was correlated with the patient's age, so the findings in Figure 4a resemble those displayed in Figure 4b.

Regarding the pathogenesis of pinguecula, its grade was higher in SCL and HCL wearers than in nonwearers, suggesting that pinguecula related to SCLs and HCLs may share a common pathogenesis. CLs have an adverse influence on tear film stability, causing break-up and thinning of the film, and increasing evaporative tear loss, probably by a disruptive effect on the tear lipid layer. ${ }^{10,11}$ Although the possibility exists that conjunctival stimulations including CL-related dryness of the eyes and the mechanical friction between CL and conjunctiva may induce pinguecula in CL wearers, the relation between CL and pinguecula is still unclear. Further investigation will be required to assess the relationship between wearing CLs and the pathogenesis of pinguecula. 
a
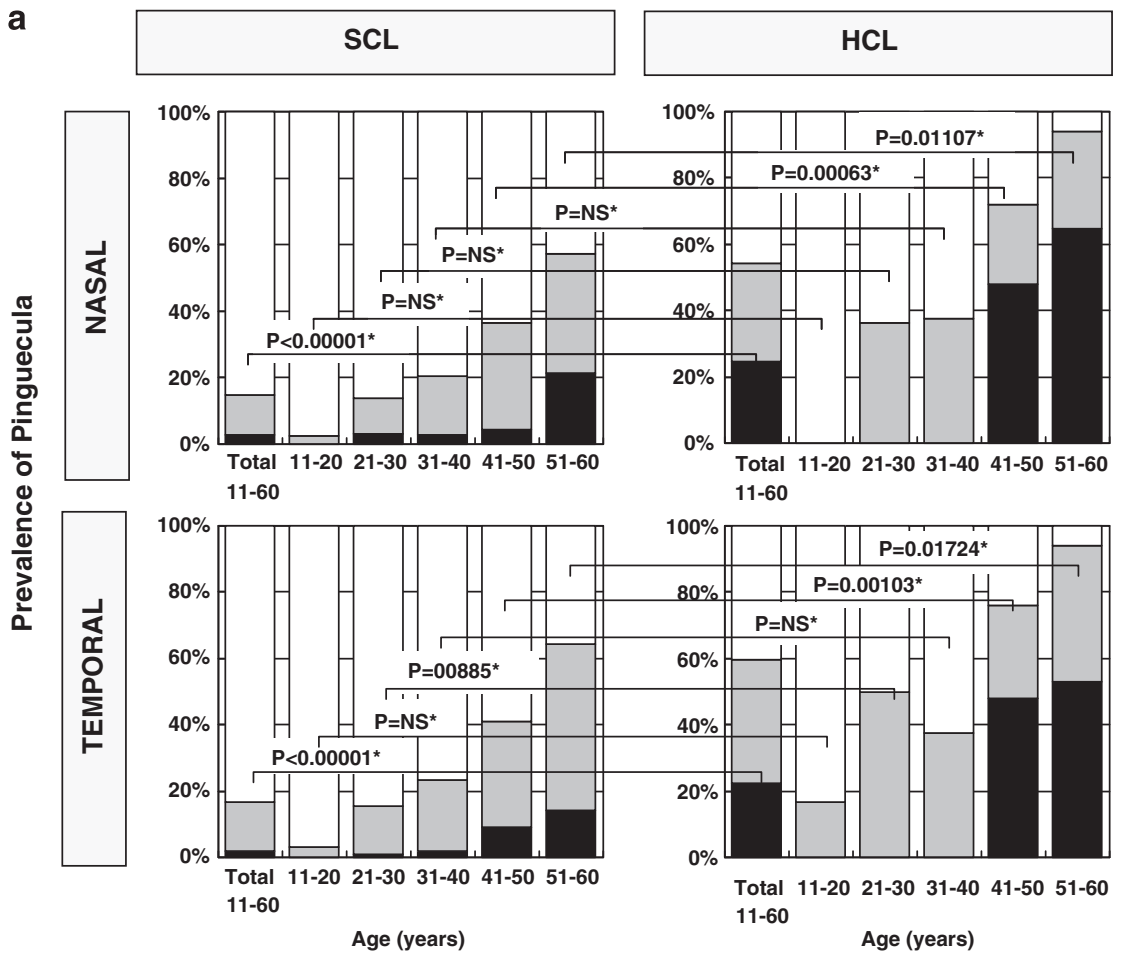

b
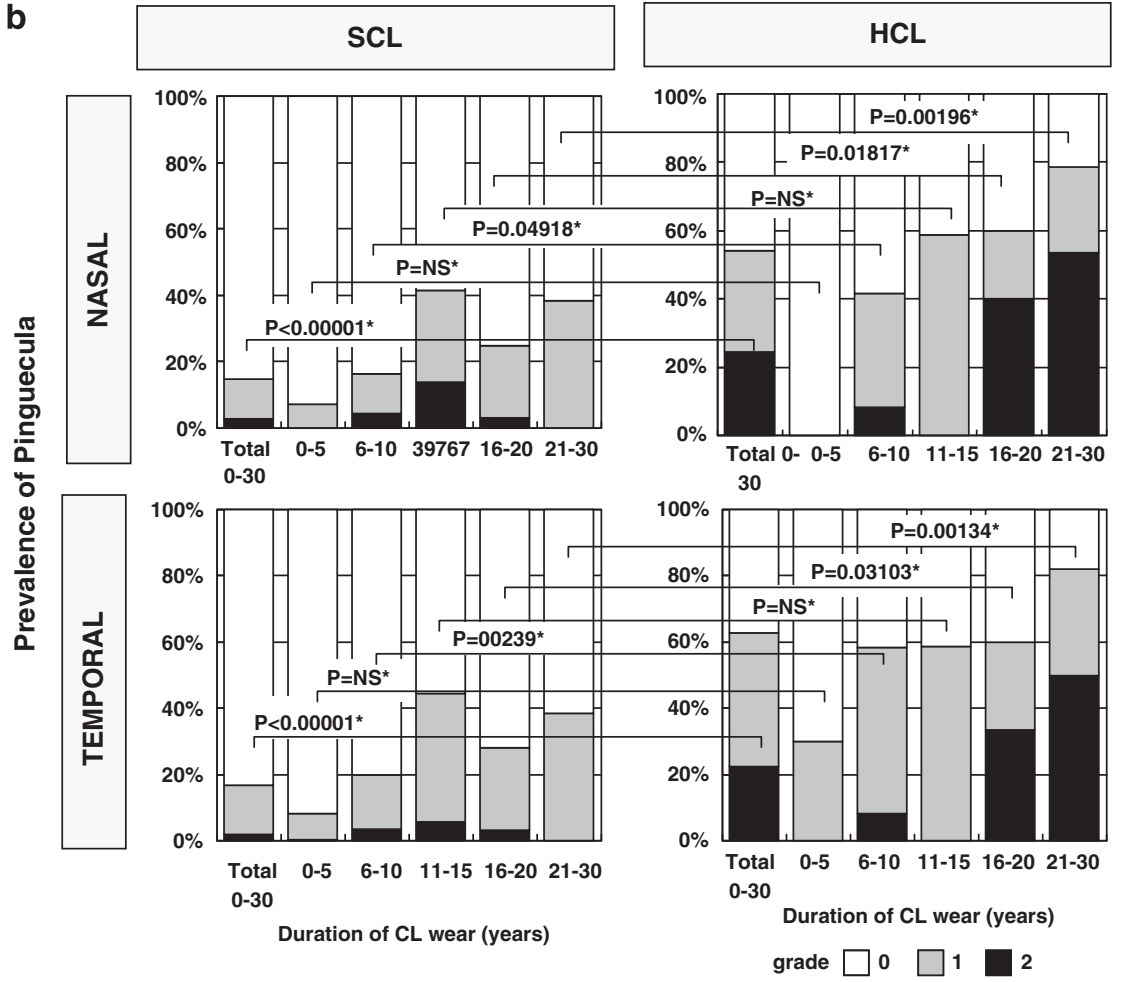

Figure 4 Comparison of the grade of pinguecula in each age group (a) and duration of CL wear (b) between SCL and HCL wearers. *Mann-Whitney $U$-test. Abbreviation: NS = not significant.

Despite the larger size of SCLs, the grade of pinguecula in SCL wearers was lower than in HCL wearers

(Figures $4 \mathrm{a}$ and $\mathrm{b}$ ). There may be several reasons for this.
First, other features of a CL, such as its edge, curvature, elasticity, thickness, and fit, may be more important for inducing conjunctival stimulation than its size. 


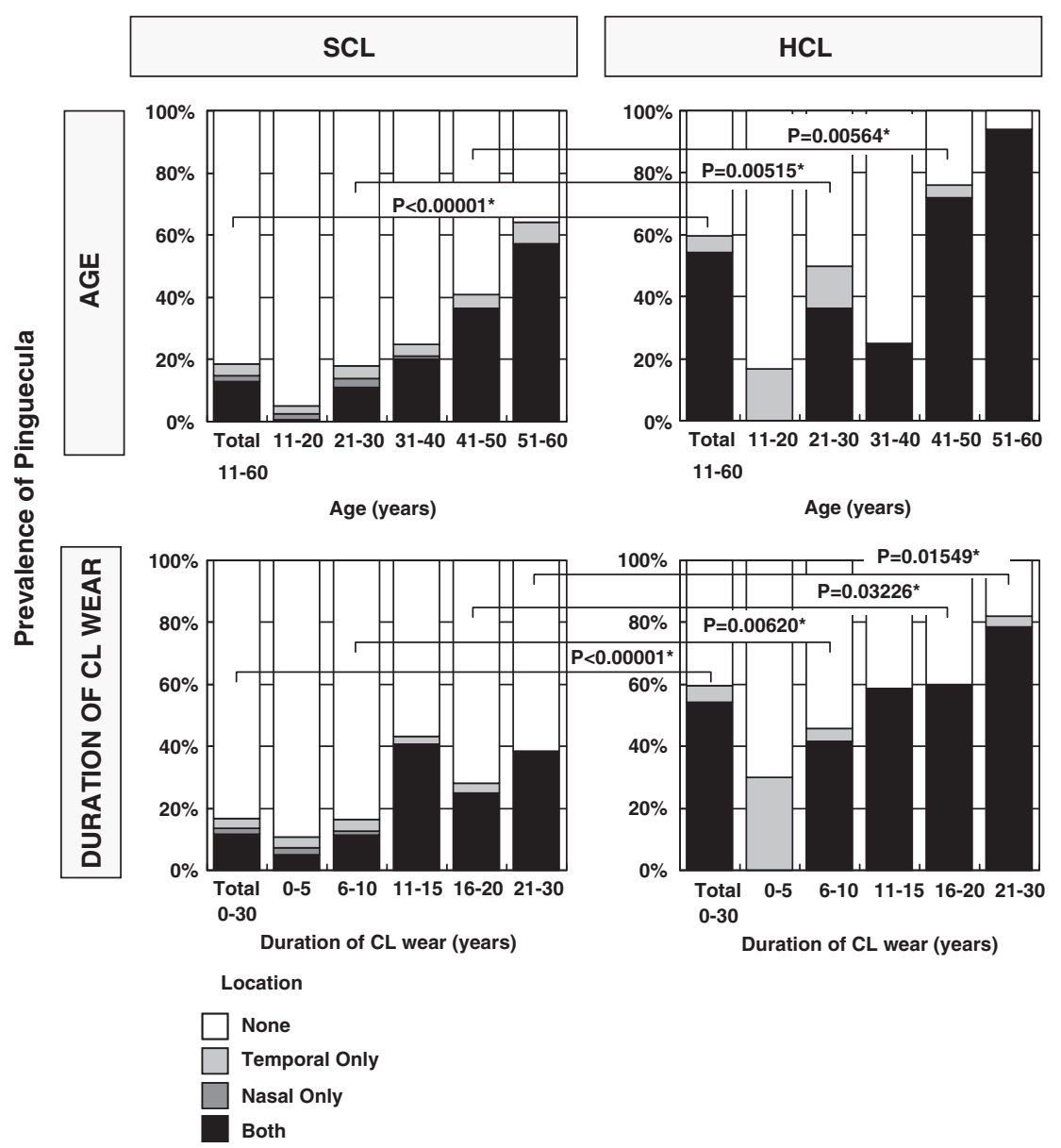

Figure 5 Comparison of the location of pinguecula in each age group and duration of CL wear between SCL and HCL wearers. The location of pingueculal was classified into four categories: (1) no pinguecula, (2) temporal pinguecula only, (3) nasal pinguecula only, and (4) both temporal and nasal pinguecula. *Mann-Whitney $U$-test.

HCLs may cause more severe chronic stimulation of the conjunctiva than SCLs because of their greater stiffness and larger elastic modulus. Second, SCLs have a larger diameter than HCLs and thus provide limbal protection against ultraviolet radiation (UVR), whereas HCLs with a smaller diameter provide less protection for the limbal cornea and leave the limbal conjunctiva exposed to UVR. ${ }^{12,13}$ The newer silicone hydrogel SCLs with greater oxygen permeability than conventional SCLs are thought to reduce the risk of hypoxia affecting the limbal epithelium and conjunctiva. ${ }^{14}$ Furthermore, SCLs are larger and softer, so that these lenses can cover pinguecula without causing rubbing, whereas the firm edge of HCLs stimulates both the conjunctiva and any pinguecula that exists.

This study had several limitations. One major drawback is that hospital-based studies tend to assess a selected sample of the population with different risk factors. Second, the cross-sectional sample is also influenced by selection and cannot indicate the CL-induced changes of pinguecula. Long-term follow-up of the same patients will be needed to achieve this. Third, although keratoconjunctivitis sicca may be responsible for pinguecula, we did not evaluate dry eye symptoms. Despite these limitations, this study demonstrated the relationship between CL wearing and the severity of pinguecula. CL physicians need to inform the patients of the longer term cosmetic from an increased risk of pingencula.

In summary, we reported the first assessment of the grade of pinguecula in CL wearers. We found that the prevalence and severity of pinguecula were dependent on the age and the duration of wearing CLs. The grade of pinguecula was highest in HCL wearers, followed SCL wearers, and non-wearers. These results indicate that pinguecula could be one of the complications related to wearing CLs. 


\section{Summary}

\section{What was known before}

- Pinguecula is a relatively frequent disease; however, there have been no reports about the grade of pinguecula in contact lens (CL) wearers.

\section{What this study adds}

- The grade of pinguecula was higher in CL wearers than in the non-wearers, whereas it was higher in hard CL wearers than soft CL wearers. Our results suggest that the use of CLs is an important risk factor for pinguecula.

\section{Conflict of interest}

The authors declare no conflict of interest.

\section{Acknowledgements}

We thank Dr Norihiko Yokoi of the Kyoto Prefectural University of Medicine, for helpful discussions and suggestions.

\section{References}

1 Archila EA, Arenas MC. Etiopathology of pinguecula and pterigium. Cornea 1995; 14: 543-544.

2 Panchapakesan J, Hourihan F, Mitchell P. Prevalence of pterygium and pinguecula: the Blue Mountains Eye Study. Aust N Z J Ophthalmol 1998; 26(Suppl 1): S2-S5.
3 Lim R, Mitchell P, Cumming RG. Cataract associations with pinguecula and pterygium: the Blue Mountains Eye Study. Am J Ophthalmol 1998; 126: 717-719.

4 Pham TQ, Wang JJ, Rochtchina E, Mitchell P. Pterygium/ pinguecula and the five-year incidence of age-related maculopathy. Am J Ophthalmol 2005; 139: 536-537.

5 Pham TQ, Wang JJ, Rochtchina E, Mitchell P. Pterygium, pinguecula, and 5-year incidence of cataract. Am J Ophthalmol 2005; 139: 1126-1128.

6 Fotouhi A, Hashemi H, Khabazkhoob M, Mohammad K. Prevalence and risk factors of pterygium and pinguecula: the Tehran Eye Study. Eye 2009; 23: 1125-1129.

7 Mimura T, Yamagami S, Usui T, Funatsu H, Mimura Y, Noma $\mathrm{H}$ et al. Changes of conjunctivochalasis with age in a hospital-based study. Am J Ophthalmol 2009; 147: 171-177.

8 Mimura T, Usui T, Yamamoto H, Yamagami S, Funatsu H, Noma $\mathrm{H}$ et al. Conjunctivochalasis and Contact Lenses. Am J Ophthalmol 2009; 148: 20-25.

9 Holden BA. The Glenn A. Fry Award lecture 1988: the ocular response to contact lens wear. Optom Vis Sci 1989; 66: 717-733.

10 Holden BA, Sweeney DF, Seger RG. Epithelial erosions caused by thin high water content lenses. Clin Exp Optom 1986; 69: 103-107.

11 Guillon JP. Tear film structure on contact lenses. In: Holly FJ (ed). The Preocular Tear Film in Health, Disease, and Contact Lens Wear. Dry Eye Institute: Lubbock (TX), 1986, pp 914-919.

12 Bergmanson JP, Sheldon TM. Ultraviolet radiation revisited. CLAO J 1997; 23: 196-204.

13 Harris MG, Haririfar M, Hirano KY. Transmittance of tinted and UV-blocking disposable contact lenses. Optom Vis Sci 1999; 76: 177-180.

14 Court JL, Redman RP, Wang JH, Leppard SW, Obyrne VJ, Small SA et al. A novel phosphorylcholine-coated contact lens for extended wear use. Biomaterials 2001; 22: 3261-3272. 